\title{
Venous thromboembolism
}

\section{prophylaxis: a path toward more appropriate use}

\section{Paul J Grant, Scott A Flanders}

Division of General Medicine, Department of Internal Medicine, University of Michigan Health System, Ann Arbor, Michigan, USA

\section{Correspondence to}

Dr Paul J Grant, Division of General Medicine, Department of Internal Medicine, University of Michigan Health System,

3119 Taubman Center, 1500 E. Medical Center Dr., P.O. Box 5376, Ann Arbor, MI 48109, USA; paulgran@med.umich.edu

Accepted 17 July 2015 Published Online First 5 August 2015

\section{SLinked}

- http://dx.doi.org/10.1136/ bmjqs-2015-003992

\section{CrossMark}

\author{
To cite: Grant PJ, \\ Flanders SA. BMJ Qual Saf \\ 2015:24:605-607.
}

Hospital-acquired venous thromboembolism (VTE) is a leading cause of preventable death in hospitalised patients and its prevention with pharmacological prophylaxis has been rated a top patient safety practice. ${ }^{1}$ Furthermore, the rate at which VTE prophylaxis is administered to 'at-risk' patients along with the rate of 'potentially preventable' VTE events are national performance measures for US hospitals. ${ }^{2}$ As a result, many hospitals have spent considerable time and effort implementing processes designed to increase rates of VTE prophylaxis. Missed doses due to patient refusal of VTE prophylaxis is a commonly encountered barrier. Strategies to minimise anticoagulant refusal in patients who would otherwise benefit from prophylaxis are needed.

Baillie $e t a l^{3}$ describe a multifaceted approach designed to increase adherence to pharmacological VTE prophylaxis in hospitalised patients. By standardising the nursing response to patient refusal of heparin injections (which included a strong focus on patient education), assessing successful administration of VTE prophylaxis on a daily basis via a multidisciplinary rounding checklist, and receiving regular feedback on patient refusal rates, the authors were able to demonstrate a reduction in missed doses in over 20000 patient admissions to medical and oncology units. Much of the improvement was due to reductions in patient refusal. The fact that the reduction in missed doses was not seen in several units serving as the control group further supports the effectiveness of the intervention.

As the authors highlight, which aspects of the multifaceted intervention primarily influenced the improvement remains unknown. For example, the intervention's nursing response to patient refusal included offering low molecular weight heparin
(LMWH) as an alternative to unfractionated heparin (LMWH requires fewer injections but is not the default due to higher costs). The post-intervention cohort saw a fivefold increase in the use of LMWH, which likely contributed to the significant drop in the percentage of patients missing any dose. However, there was also an increase in administered prophylaxis doses (as a percentage of the total hospital stay) to patients who initially refused, suggesting the benefit did not solely come from increased LMWH use. The patient education efforts probably also contributed to the improvement. Although the authors successfully improved adherence to orders for VTE prophylaxis, the study had insufficient power to detect a difference in hospital-acquired VTE event rates. We are thus left without a clear understanding of the intervention's impact on patient outcomes. But are improved clinical outcomes necessary to demonstrate that this intervention benefited patients? One might argue that decreasing rates of refusal and increasing prophylaxis rates for all hospitalised patients is good enough. We are not so sure.

Many thought leaders argue that the vast majority of hospitalised patients have significant risk factors for VTE. Since large randomised controlled trials have shown a profound reduction in deep vein thrombosis (DVT) and pulmonary embolism (PE) events (albeit including asymptomatic events in highly selected patient populations), ${ }^{4-6}$ experts increasingly advocated for the implementation of widespread prophylaxis strategies for almost all hospitalised patients. In recent years, however, we have learned that the observed rate of symptomatic VTE in hospitalised medical patients is actually much lower than previously suggested, varying between $0.4 \%$ and $1.0 \%$ in several series. ${ }^{78}$ Our own work with the 
Michigan Hospital Medicine Safety Consortium, a clinical quality collaborative and data registry consisting of over 100000 hospitalised medical patients from 48 hospitals, has also demonstrated a low VTE event rate in non-ICU medical patients.

Furthermore, using a definition of risk that considers most hospitalised medical patients at elevated risk for VTE, we have shown that hospitals using pharmacological prophylaxis in a high percentage of their patients did not have lower severity adjusted rates of VTE when compared to hospitals which used prophylaxis more sparingly. ${ }^{9}$ The percentage of patients receiving pharmacological prophylaxis at the high utilisation hospitals in the Michigan collaborative (84\%) was comparable to the rate of prophylaxis in the study by Baillie $e t a l^{3}$ (prophylaxis ordered for $82 \%$ of the length of stay). This suggests that without any convincing data showing an impact on VTE outcomes, or at least a better understanding of the VTE risk profile for patients included in the Baillie $e t a l^{3}$ study, we are unsure if the increasing rates of prophylaxis led to a net benefit.

There are high-risk cohorts of patients who should routinely receive VTE prophylaxis. For example, patients hospitalised with cancer are repeatedly shown to be at particularly high risk for VTE. We would argue that the strategies employed by Baillie $e t a^{3}$ to broadly increase prophylaxis in this cohort were well targeted. However, it is increasingly evident that not all hospitalised medical patients require pharmacological prophylaxis. ${ }^{7}$ Depending on the risk assessment model used, the size of the population at low risk for VTE may be as large as $60 \%$ or more for non-ICU medical patients. ${ }^{10}$ In low-risk patients, it is unlikely that pharmacological prophylaxis will offer any benefit, and the risks-which include bleeding, patient discomfort and excess costs-could be minimised by avoiding unnecessary anticoagulant therapy.

The American College of Chest Physicians strongly recommends avoiding VTE prophylaxis (pharmacological and mechanical) in low-risk medical patients in their most recent clinical practice guideline. ${ }^{11}$ In our Michigan collaborative, avoidance of pharmacological prophylaxis in low-risk patients (by any definition) is seldom practiced, perhaps due to years of hearing that most patients are at risk for VTE and warrant prophylaxis. We are now focusing on identifying strategies our hospitals can use to decrease VTE prophylaxis administration in low-risk patients.

This need for 'undiffusion' of established practice $^{12}$ was recently described in reference to numerous clinical practices where newer data highlight a need to eliminate practices we once thought were beneficial (eg, perioperative $\beta$-blockers, stress-dosed steroids in the ICU, postoperative blood transfusions, and tight blood glucose control). These are all examples where we have come to recognise that 'less is more' and it seems that pharmacological VTE prophylaxis in the subset of lower risk medical patients is yet another example. As our knowledge in this field improves, the pendulum needs to swing back away from the common practice of VTE prophylaxis for all patients.

In addition to efforts to reduce the rate of VTE prophylaxis for low-risk patients while ensuring delivery for the high-risk subsets, the challenge remains over what to do with the large moderate-risk group. Individualised risk assessment for both VTE and bleeding may be able to distinguish which patients would derive more benefit than risk from anticoagulant therapy. Although physicians and quality leaders have historically felt most comfortable using guidelines, checklists and automated order sets to deliver care, this may be an area where individualised patientcentred care and shared decision-making will drive the future direction of VTE prophylaxis. Engaging patients in the discussion about prophylaxis use, as was effectively done by Baillie et $a l,{ }^{3}$ will be critical if we are to succeed. William Osler once said "medicine is a science of uncertainty and an art of probability". As it is almost certain that a perfect VTE risk assessment model will never be developed, this sentiment will surely remain true.

Competing interests None declared.

Provenance and peer review Commissioned; internally peer reviewed.

\section{REFERENCES}

1 Shekelle PG, Pronovost PJ, Wachter RM, et al. The top patient safety strategies that can be encouraged for adoption now. Ann Intern Med 2013;158:365-8.

2 The Joint Commission. Venous thromboembolism core measure set. http://www.jointcommission.org/core_measure_ sets.aspx (accessed 10 Jul 2015).

3 Baillie CA, Guevara JP, Boston RC, et al. A unit-based intervention aimed at improving patient adherence to pharmacological thromboprophylaxis. BMJ Qual Saf 2015; 24:654-60.

4 Samama MM, Cohen AT, Darmon J, et al. A comparison of enoxaparin with placebo for the prevention of venous thromboembolism in acutely ill medical patients. New Engl J Med 1999;341:793-800.

5 Leizorovicz A, Cohen AT, Turpie AGG, et al. Randomized, placebo-controlled trial of dalteparin for the prevention of venous thromboembolism in acutely ill medical patients. Circulation 2004;110:874-9.

6 Cohen AT, Davidson BL, Gallus AS, et al. Efficacy and safety of fondaparinux for the prevention of venous thromboembolism in older acute medical patients: randomized placebo controlled trial. BMJ 2006;332:325-9.

7 Lederle FA, Zylla D, MacDonald R, et al. Venous thromboembolism prophylaxis in hospitalized medical patients and those with stroke: a background review for an American College of Physicians Clinical Practice Guideline. Ann Intern Med 2011;155:602-15. 
8 Rothberg MB, Lindenauer PK, Lahti M, et al. Risk factor model to predict venous thromboembolism in hospitalized medical patients. J Hosp Med 2011;6:202-9.

9 Flanders SA, Greene MT, Grant P, et al. Hospital performance for pharmacologic venous thromboembolism prophylaxis and rate of venous thromboembolism: a cohort study. JAMA Intern Med 2014;174:1577-84.

10 Barbar S, Noventa F, Rossetto V, et al. A risk assessment model for the identification of hospitalized medical patients at risk for venous thromboembolism: the Padua Prediction Score. J Thromb Haemost 2010;8:2450-7.

11 Guyatt G, Akl EA, Crowther M, et al. Introduction to the 9th edition: Antithrombotic Therapy and Prevention of Thrombosis, 9th ed: American College of Chest Physicians Evidence-Based Clinical Practice Guidelines. Chest 2012;141(2 Suppl):48S-52S.

12 Davidoff F. On the undiffusion of established practices. JAMA Intern Med 2015;175(5):809-11. 